\title{
Expression of peroxiredoxins in the human testis, epididymis and spermatozoa and their role in preventing $\mathrm{H}_{2} \mathrm{O}_{2}$-induced damage to spermatozoa
}

\author{
Hui Shi ${ }^{1 \dagger}$, Juan Liu' ${ }^{2 \dagger}$, Peng Zhu², Haiyan Wang², Zhenjun Zhao', \\ Guohong Sun ${ }^{3}$, Jianyuan $\mathrm{Li}^{1}{ }^{\text {, }}$
}

${ }^{1}$ College of Life Science, Yantai University (Yantai, Shandong Province, 264005 PR China)

${ }^{2}$ Yantai Yuhuangding Hospital (Yantai, Shandong Province, 264000, PR China)

${ }^{3}$ Yantai Vocational College (Yantai, Shandong Province, 264670, PR China)

${ }^{4}$ Key Laboratory of Male Reproductive Health, National Health and Family Planning Commission (Beijing, 100081, PR China)

†These authors contributed equally to the work presented here.

\begin{abstract}
Introduction. High levels of reactive oxygen species (ROS) have potential toxic effects on testicular function and sperm quality. Peroxiredoxins (PRDXs) are enzymes with a role as ROS scavenger. The aim of the study was to reveal the presence and localization of PRDXs in human testis, epididymis and spermatozoa, and the protective roles of PRDX2 and PRDX6 in sperm motility.

Material and methods. The presence and localization of PRDXs in the human testis, epididymis and spermatozoa were detected by immunohistochemistry, western blot and immunofluorescence. The effect of anti-peroxidative damage to spermatozoa was examined by adding $\mathrm{H}_{2} \mathrm{O}_{2}$ to the recombinant protein-treated spermatozoa.

Results. There were strong signals of PRDX1 in spermatogonia and round spermatids; PRDX2 in the round spermatids; PRDX4 and 5 in spermatogonia; PRDX6 in Sertoli cells. PRDXs were also found in epididymal epithelial cells where the expression of PRDX1, 4, 5, 6 in the cauda was higher than in the caput of epididymis. PRDX1-6 immunoreactivity was found throughout acrosome, post-acrosomal region, equatorial segment, neck and cytoplasmic droplet, midpiece and principal piece. The $\mathrm{H}_{2} \mathrm{O}_{2}$-induced reduction in sperm motility was reversed by recombinant PRDX2 or PRDX6 in a dose-dependent manner.

Conclusions. PRDX1-6 in the human testis and epididymis presented cell-specificity. PRDX2 and 6 are potential antioxidant protectors for human spermatozoa. (Folia Histochemica et Cytobiologica 2018, Vol. 56, No. 3, 141-150)
\end{abstract}

Key words: Peroxiredoxins; human; testis; epididymis; spermatozoa

\section{Introduction}

Reactive oxygen species (ROS), acting as both toxic and physiological molecules in spermatozoa $[1,2]$, are formed as natural products of the metabolism

Correspondence address: Jianyuan $\mathrm{Li}$

Yantai University, No. 30 Qingquan Road,

Yantai, 264005, P. R. CHINA

phone: 86-535-6902638

e-mail: shihuineau@163.com; 201303000002@ytu.edu.cn of oxygen. Small amounts of ROS are necessary for spermatozoa to acquire their fertilizing capability in the female tract [2,3]. However, in the male reproductive system, the generation of ROS at high levels has potential toxic effects on testicular function and sperm quality and function $[4,5]$. High seminal ROS levels may be detected in $25 \%$ to $40 \%$ of infertile men [6].

Many antioxidant enzymes are present in different parts of the male reproductive tract, such as superoxide dismutase (SOD), catalase (CAT) and glutathione peroxidase (GPX), which also exist in 
semen. Moreover, several non-enzymatic antioxidants (e.g. vitamins $\mathrm{C}$ and $\mathrm{E}$, hypotaurine, taurine, L-carnitine,) are also found in semen. Over the past few years, an important enzymatic antioxidant protein family, the peroxiredoxins (PRDXs), which association with infertility was first reported by Gong et al. (2012) [7], has been associated with reproduction. PRDXs comprise up to $1 \%$ of soluble cellular proteins $[8,9]$ which suggests that PRDXs have a potential important role in the response of cells to oxidative stress. Mammals have six PRDXs, with PRDX1, 2 and 6 found in the cytoplasm, PRDX4 in the endoplasmic reticulum, PRDX3 in the mitochondria, and PRDX5 in various compartments of the somatic cell, including peroxisomes and mitochondria [10]. Abnormal function of PRDXs is associated with male infertility [11]. In particular, an extensive literature search revealed that PRDXs are important for sperm function and male fertility [12-16].

Although the role of PRDXs in reproductive system had been to some extent reported, the functions of PRDXs, especially in humans, remain to be revealed. In general, there were close connection between locations and functions of proteins. To date, the localizations of PRDXs in the testis, epididymis and mature spermatozoa have been described in mouse, rat and boar [17-21] but not in humans. As the testes and epididymides provide the environment for spermatogenesis, sperm maturation and storage, we examined the PRDXs in human testis and epididymis for the first time, and determined their role in combating hydrogen peroxide-induced damage to sperm motility.

\section{Material and Methods}

Materials. Rabbit polyclonal anti-PRDX1 (PAB8052) and mouse polyclonal anti-PRDX3 (H00010935-B01P) were purchased from Abnova (Taipei, Taiwan). Goat polyclonal anti-PRDX2 (sc-23967) was purchased from Santa Cruz Biotechnology (CA, USA). Mouse monoclonal anti-PRDX4 (ab16943) and anti-PRDX5 (ab16944) were purchased from Abcam Inc (Cambridge, MA, USA). Rabbit polyclonal anti-PRDX6 was prepared inhouse as described below. FITC-labeled rabbit anti-goat $\operatorname{IgG}(\mathrm{AP} 106 \mathrm{~F})$ and goat anti-rabbit IgG (AP132F) were purchased from Chemicon (Teme Cula, CA, USA). FITC-labeled goat anti-mouse IgG (ZF-0312), horseradish peroxidase (HRP)-conjugated goat anti-rabbit IgG (ZB-2301), goat anti-mouse IgG (ZB-2305), rabbit anti-goat $\operatorname{IgG}(\mathrm{ZB}-2306)$ and a diaminobenzidine (DAB) kit (ZLI-9033) were purchased from ZSGB-BIO (Beijing, China). Propidium iodide was purchased from Invitrogen (Carlsbad, CA, USA). The pET-32b (+) vector, pET-44b
$(+)$ vector and E. coli BL21 (DE3) were purchased from Novagen (Madison, WI, USA). Polyvinylidene difluoride (PVDF) membrane (immune-blot) was purchased from GE Healthcare (Piscataway, NJ, USA). Other chemicals were at least of reagent grade.

Preparation of PRDX6 antiserum. The purity and activity of PRDX6 recombination protein, used for antiserum preparation, were confirmed previously [22]. A basic immunization was done by injecting into a New Zealand rabbit $100 \mu \mathrm{g}$ of PRDX6 recombination protein, emulsified with $1 \mathrm{ml}$ of Freund's complete adjuvant (RC-s1, Sigma, USA). Two successive boosts were carried out with an interval of 2 weeks after the first immunization. For each boost, $100 \mu \mathrm{g}$ of PRDX6 and $1 \mathrm{ml}$ of Freund's incomplete adjuvant were used. For the fourth immunization, $200 \mu \mathrm{g}$ of PRDX6 and $1 \mathrm{ml}$ of Freund's incomplete adjuvant were used. Antiserum was collected $7 \mathrm{~d}$ after the last boost.

The specificity of the antiserum was confirmed by immunoblot with the commercial full-length human PRDX6 recombinant protein (LF-P0004, Invitrogen, Carlsbad, CA, USA), which was without the tag protein.

Human samples. The testes and epididymides from three accident victims aged 31, 33 and 34 years were obtained from Yantai Yuhuangding Hospital, Yantai, China. These men had records documenting a history of fatherhood and no disease of the reproductive system. Their family members supplied written approval for the use of these organs for research. Twenty healthy volunteers from 25 to 30 years of age donated human spermatozoa by masturbation after 7 days of sexual abstinence. The quality of the semen used for immunofluorescence studies was determined on the basis of sperm concentration, motility and morphology. Only semen samples that met the WHO $(1999,2010)$ values were used. All the human organ collection procedures were approved by the Ethics Committee of Yantai Yuhuangding Hospital.

Protein extraction and western blot. Human testicular and epididymal proteins were extracted according to the methods of Lin et al. (2008) [23]. Human semen was allowed to liquefy for $30 \mathrm{~min}$ at $37^{\circ} \mathrm{C}$ before the gentle addition of phosphate-buffered saline (PBS: $20 \mathrm{mmol} / \mathrm{l} \mathrm{PB}, 0.15 \mathrm{~mol} / \mathrm{l}$ $\mathrm{NaCl}, \mathrm{pH}$ 7.2) on top to allow spermatozoa to swim up for $1 \mathrm{~h}$. Spermatozoa ( $10^{7}$ cells) were mixed with loading buffer and boiled for $5 \mathrm{~min}$. The proteins were separated by $12 \%$ (w/v) SDS-PAGE and transferred to a PVDF membrane. After incubation in blocking buffer (PBS with 5\% (w/v) nonfat powdered milk) for $1 \mathrm{~h}$ at room temperature, membranes were first immunoblotted with the related anti-PRDX antibodies overnight at $4^{\circ} \mathrm{C}$. Related HRP-conjugated second antibody and the DAB kit (ZSGB-BIO, Beijing, China) were used for visualization. The result given is one representative triplicate. 
Immunohistochemical staining. Human testicular and epididymal tissues were immersed in Bouin's fixative for $24 \mathrm{~h}$, followed by standard paraffin embedding procedures. $4-\mu \mathrm{m}$ sections were deparaffinized, rehydrated and antigens unmasked in a microwave oven for $20 \mathrm{~min}$ [24]. After quenching of endogenous peroxidase activity with $3 \%(\mathrm{v} / \mathrm{v})$ hydrogen peroxide for $10 \mathrm{~min}$, the hybridization of sections were performed according to the similar procedures with western blot. The peroxidase activity was revealed by DAB kit (ZSGB-BIO, Beijing, China). After counterstaining of slides with hematoxylin, the sections were examined with a bright-field microscopy (DM LB2 microscope, Leica, Nussloch, Germany). The result given is one representative triplicate.

Indirect immunofluorescence of ejaculated spermatozoa. Spermatozoa, washed three times with PBS, were placed on $1 \%(\mathrm{w} / \mathrm{v})$ gelatin-coated slides, air-dried and fixed with ice-cold $100 \%$ (v/v) methanol for $10 \mathrm{~min}$. The slides were blocked for $1 \mathrm{~h}$ at room temperature with $3 \%(\mathrm{w} / \mathrm{v}) \mathrm{BSA}$ in PBS and incubated with the relevant anti-PRDXs antibodies (diluted 1:50 in blocking solution) overnight at $4^{\circ} \mathrm{C}$. Finally, sections were washed with PBS-Tween (1000:1) and incubated with a 1:200 dilution of FITC-labeled goat anti-mouse IgG (for PRDX3, 4, 5), rabbit anti-goat IgG (for PRDX2) or goat anti-rabbit IgG (for PRDX1, 6) for 1 $\mathrm{h}$ at room temperature. Propidium iodide $(0.01 \mathrm{mg} / \mathrm{ml})$ was added for counterstaining the nuclei after incubation. The slides were washed three times with PBS-Tween, mounted in $80 \%(\mathrm{v} / \mathrm{v})$ glycerol and examined with a Meta 510 laser scanning microscope (Zeiss, Jena, Germany). Positive control was HEL-S-25a, demonstrated in a previous study [25]. The experiment was conducted for four times and involved five donors for each time.

Preparation of PRDX2 and PRDX6 proteins. The $P R D X 2$ and $P R D X 6$ genes were directly amplified by PCR from our human epididymal cDNA library [23] with the specific primers (PRDX2-F: 5' - gtaccatatggcctccggtaacgcgc-3; PRDX2-R: 5'-ttactcgagattgtgtttggagaaatattc-3') and (PRDX6-F: 5'-tatccatatgcceggaggtctgcttc-3; $P R D X 6$-R: 5'-ttactcgagaggctggggtgtgtagcg-3'), each containing NdeI and $X h o$ I sites. The PCR products (full length of $P R D X 2$ and $P R D X 6$ ) were digested with $N d e \mathrm{I}$ and $X h o \mathrm{I}$, and inserted into pET-44b (+) and pET-32b (+) vectors, respectively. The plasmids of $\mathrm{pET}-44 \mathrm{~b}$ $(+) / P R D X 2$ and pET-32b $(+) / P R D X 6$ were transformed in E. coli BL21 (DE3) strain (Novagen, Madison, WI, USA) according to the supplier's instructions. The transformed $E$. coli were grown to mid-log phase and fusion protein expression was induced with $1 \mathrm{mM}$ isopropyl-1-thio-D-galactoside (IPTG) for $3 \mathrm{~h}$ at $32^{\circ} \mathrm{C}$. The cells were sonicated and the fractions analyzed on $15 \%(\mathrm{w} / \mathrm{v})$ polyacrylamide gels and stained with Coomassie blue G-250 according to standard procedures [26]. The histidine tag in the vector permitted the recombinant fusion protein to be purified by $\mathrm{Ni}^{2+}$-Chelating Sepharose Fast Flow resin (GE Healthcare, Piscataway, NJ, USA) according to the manufacturer's instructions. After the protein concentration was measured, the purified and endotoxin-free PRDX2 and PRDX6 proteins were conserved by freeze-drying for anti-peroxidative damage activity assays.

Investigation of PRDX2, 6 protein anti-peroxidative damage activity in vitro. Motile spermatozoa were prepared by swimup method as described above. After being washed twice with PBS, spermatozoa were incubated at a concentration of $3 \times 10^{6} / \mathrm{ml}$ in PBS buffer containing $0.5 \%(\mathrm{w} / \mathrm{v})$ BSA. The recombinant proteins (PRDX2 or PRDX6) were diluted to final protein concentrations of $0,31.25,62.5,125,250$ and $500 \mu \mathrm{g} / \mathrm{ml}$ and added to the sperm suspension before addition of $\mathrm{H}_{2} \mathrm{O}_{2}$ at a final concentration of $100 \mu \mathrm{M}$. After incubation at $37^{\circ} \mathrm{C}$ for $30 \mathrm{~min}$, sperm kinematic parameter values as indicators of sperm damage were generated by a computer-aided sperm analysis (CASA) system (HTMIVOS motility analyzer, Hamilton Thorne Biosciences, Beverly, MA, USA, Version 12.3H Build 001). The purified fusion protein induced from the pET-44b $(+)$ or pET$32 \mathrm{~b}(+)$ vector alone, without the inserted gene, was used as a negative control.

Statistical analysis. The results of the motility of spermatozoa assay (treated with recombinant or Tag proteins and $\mathrm{H}_{2} \mathrm{O}_{2}$ ) were subjected to one-way ANOVA by using JMP 4.0 software (SAS Institute, Cary, NC, USA). Results are presented as the mean + SD. Differences were considered significant at $\mathrm{P} \leq 0.05$ unless otherwise specified.

\section{Results}

\section{Western blot}

Western blot analyses showed that the rabbit polyclonal anti-PRDX6 prepared in-house recognized the commercial human PRDX6 recombinant protein specifically (Fig. 1A). PRDX1 (22 kDa), PRDX2 (22 kDa), PRDX3 (27 kDa), PRDX4 (27 kDa), PRDX5 $(17 \mathrm{kDa})$ and PRDX6 (26 kDa) proteins were expressed in the testis, epididymis and ejaculated spermatozoa. The signals of PRDX3, 4, 5 were weak in the spermatozoa (Fig. 1B).

\section{Localization of PRDXs in the human testis}

PRDX1 was highly expressed in the cytoplasm of spermatogonia and round spermatids, with a slight and homogeneous reaction present throughout the cytoplasm of the Sertoli cells and spermatocytes (Fig. 2A1). Extensive staining of PRDX2 in the nucleus, but not cytoplasm, of spermatocytes was observed. In round spermatids, intense staining was localized to the nucleus. A strong signal of PRDX2 was also present 


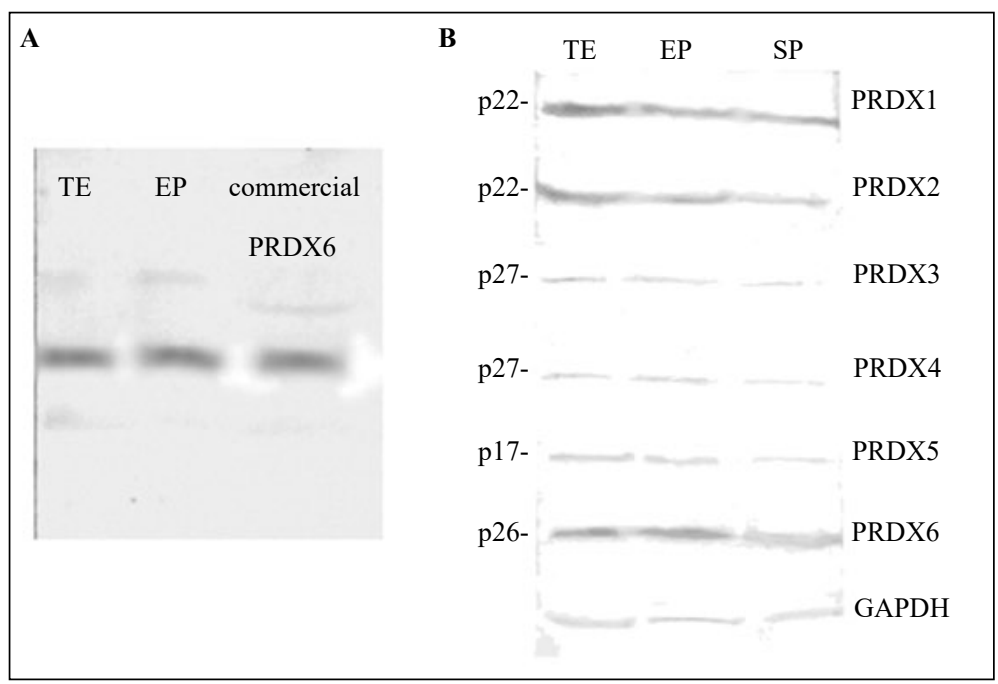

Figure 1. Specificity of the rabbit polyclonal anti-PRDX6 antibody prepared in-house (A) and expression of peroxiredoxins (PRDXs) in the human testis, epididymis and spermatozoa by western blot (B). Abbreviations: TE — testis; EP — epididymis; GAPDH - glyceraldehyde 3-phosphate dehydrogenase; SP — ejaculated spermatozoa.

in the cytoplasm of elongated spermatids (Fig. 2B1). There was a low level of PRDX3 immunoreactivity in spermatogenic cells, and only moderate staining in the cytoplasm of Sertoli cells (Fig. 2C1). Nearly the same expression of PRDX4 and PRDX5 is shown in Figs. 2D1 and 2E1. PRDX4 and PRDX5 were highly expressed in the cytoplasm of spermatogonia, with moderate staining in the cytoplasm of Sertoli cells. Specifically, the staining of PRDX4 in the residual body was detected (Fig. 2D1). Unlike the other PRDXs, the expression of PRDX6 was limited to the cytoplasm of Sertoli cells, and the spermatogonia and spermatocytes were immunonegative (Fig. 2F1). Strong expression of PRDX1, 2 and 6 was seen in Leydig cells (data not shown). A summary of the immunoreactivities of PRDXs in the human testis is presented in Table 1.

\section{Localization of PRDXs in the human epididymis}

In the caput region, there was moderate staining of PRDX1 in the principal and basal cells (Fig. 2A2). In the corpus region, the immunoreactivity of PRDX1 was observed in the principal cells, especially in their stereocilia (Fig. 2A3). Compared with the caput and corpus, strong PRDX1 staining was displayed in the stereocilia of principal cell and basal cells of the cauda region (Fig. 2A4). Although the expression of PRDX2 was limited to the principal cells throughout the epididymis, within these cells we observed a distinct intracellular compartmentalization in different regions. In the caput, the immunoreactivity was uniformly and widely in the cytoplasm of principal cells (Fig. 2B2); in the corpus and cauda, intense staining was localized to the infra-nuclear cytoplasm (Fig. 2B3) with additional strong staining in the stereocila of the cauda (Fig. 2B4). The staining of PRDX 3 in the epididymis was less prominent than that of the other PRDXs (Figs. 2C2-C4). For PRDX4, the basal cells of the caput region had no or weak staining, but there was moderately intensive staining in the principal cells (Fig. 2D2). The same cellular expression, but with stronger staining of PRDX4, was found in the corpus and cauda than caput, and also in the basal cells (Figs. 2D3-D4). Immunocytochemical staining of PRDX5 was not observed in the caput of epididymis, but the accumulation of the protein was seen in the basal cells of the corpus and cauda regions (Figs. 2E3-E4). Figure 3F shows that no PRDX6 signal was found in the caput (Fig. 2F2), and the immunoreactive signal was weak in the corpus of epididymis (Fig. 2F3) but strong in some principal and all basal cells of the cauda region (Fig. 2F4). A summary of immunoreactivities of PRDXs in the human epididymis is presented in Table 2.

\section{Localization of PRDXs in human ejaculated spermatozoa}

There was wide distribution of PRDX1 in spermatozoa with staining at the equatorial segment, post-acrosomal region and midpiece (Fig. 3A). PRDX2 was specifically detected on the anterior-acrosomal region (Fig. 3B); PRDX3 was localized at the midpiece (Fig. 3C); PRDX4 was localized to the neck (Fig. 3D), and when 


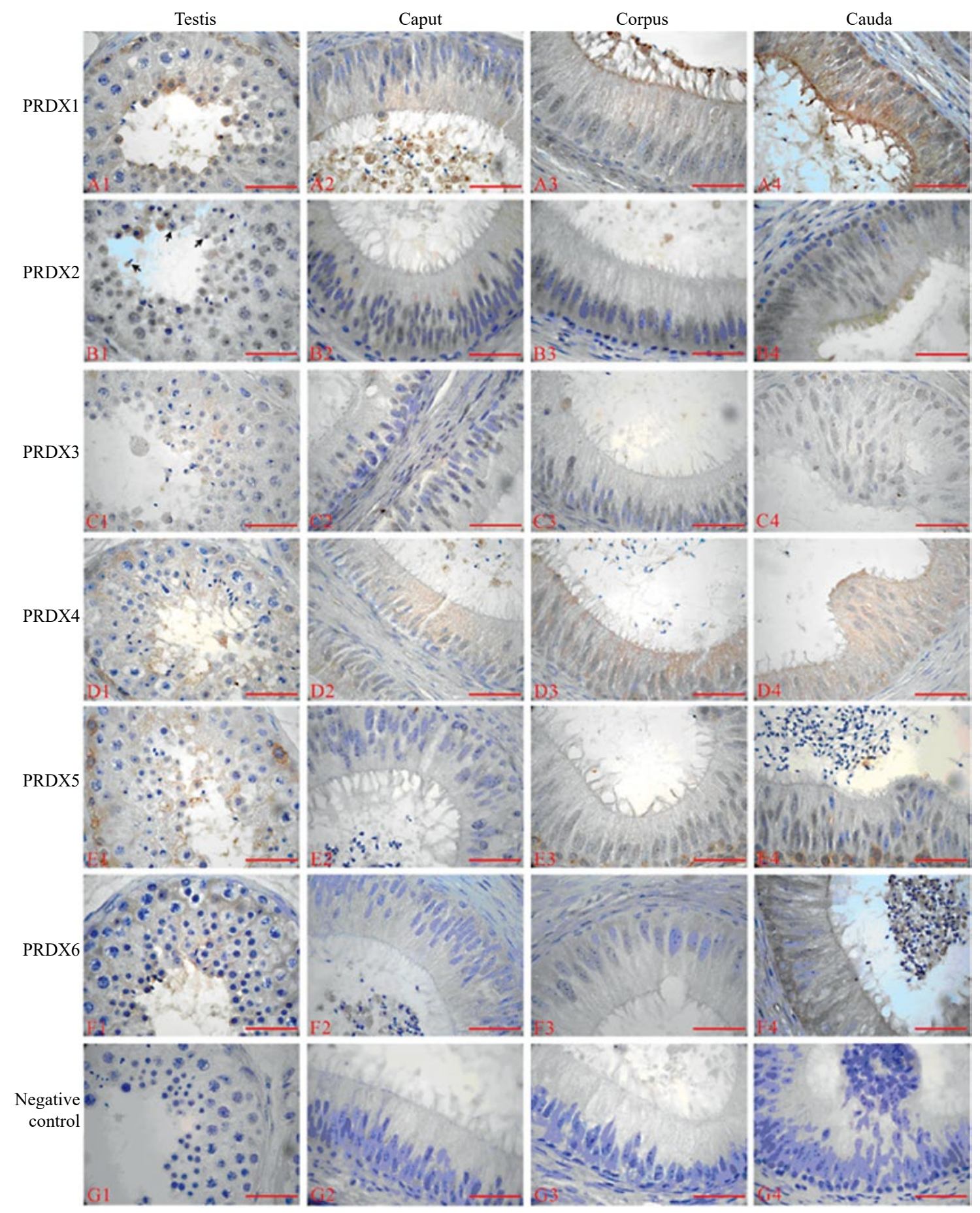

Figure 2. Immunohistochemical localization of peroxiredoxins (PRDXs) in the human testis and epididymis. The presence of PRDXs is shown in the testis (A1-F1), caput epididymis (A2-F2), corpus epididymis (A3-F3) and cauda epididymidis (A4-F4) by using anti-PRDXs antibodies. (G1-G4) represent negative control; staining specificity was checked by incubation of sections with mouse IgG (for PRDX3, 4, 5), goat IgG (for PRDX2), rabbit IgG (for PRDX1), or anti-PRDX6 antibody absorbed by its recombinant protein. The immunohistochemical stainings were performed as described in Methods. Brownish precipitates represent positive staining. Scale bars represent $40 \mu \mathrm{m}$.

a cytoplasmic droplet was present, there was intense fluorescence at that site (insert in Fig. 3D). A weak PRDX5 signal was seen on the acrosomal region
(Fig. 3E). Most staining of PRDX6 was observed on the equatorial segment, post-acrosomal region, and the principal piece, but not in the midpiece; however, 
Table 1. Intensity of the immunostaining of peroxiredoxins in the human testis

\begin{tabular}{|l|c|c|c|c|c|c|}
\hline & PRDX1 & PRDX2 & PRDX3 & PRDX4 & PRDX5 & PRDX6 \\
\hline Leydig cells & +++ & +++ & + & - & - & +++ \\
\hline Sertoli cells & + & \pm & + & + & + & +++ \\
\hline Spermatogonia & +++ & \pm & \pm & +++ & +++ & - \\
\hline Spermatocytes & + & \pm & \pm & - & + & - \\
\hline Round spermatids & +++ & +++ & \pm & \pm & + & + \\
\hline
\end{tabular}

+++ - strong staining; + - moderate staining; \pm - very weak staining; $-\longrightarrow$ no staining.
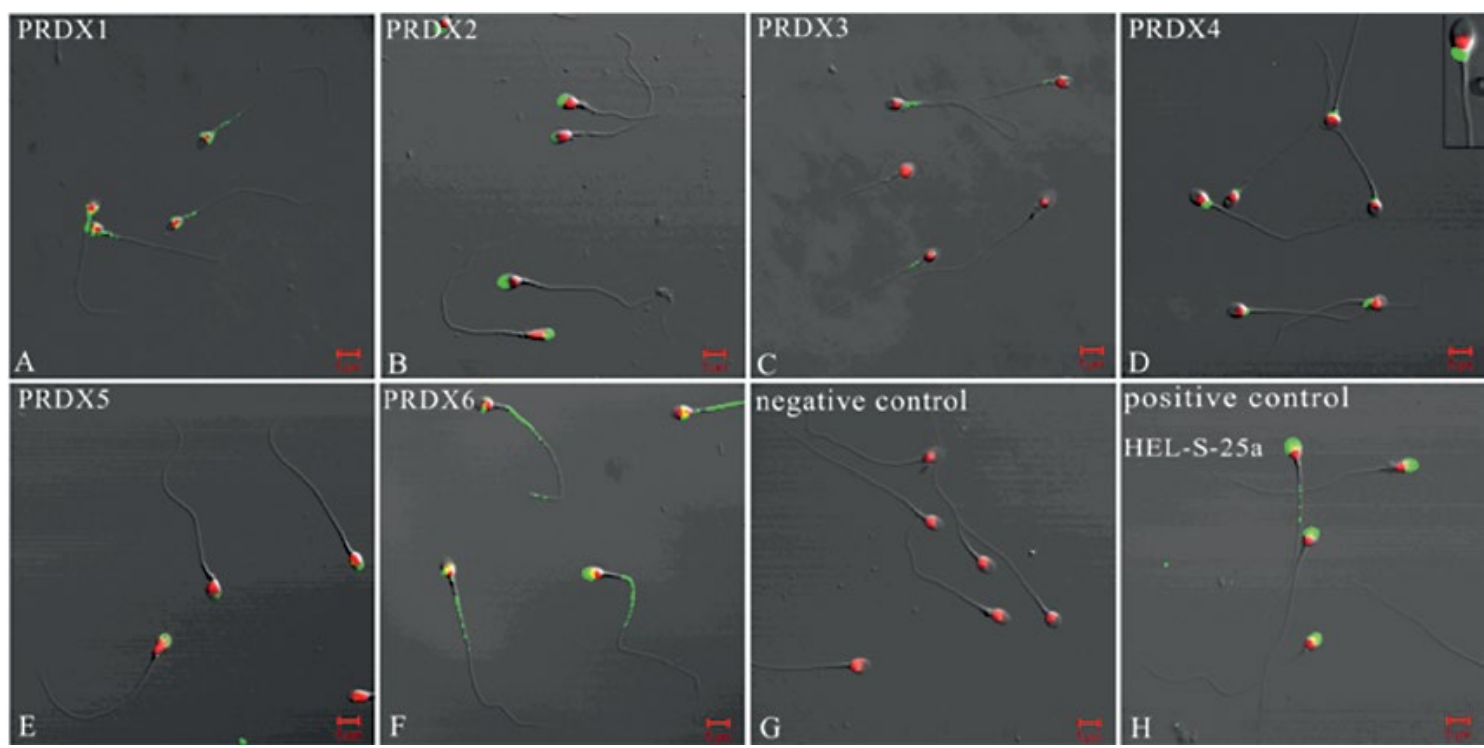

Figure 3. Immunolocalization of peroxiredoxins (PRDXs) in human ejaculated spermatozoa. The microphotographs A-F present location of PRDX1 (A), PRDX2 (B), PRDX3 (C), PRDX4 (D), PRDX5 (E) and PRDX6 (F), whereas (G) and (H) present negative and positive control, respectively. Positive control, HEL-S-25a, was a human epididymal secretory sperm-located protein. Inset in frames D is the fluorescence image of a spermatozoon with an intact cytoplasmic droplet. Scale bars represent $5 \mu \mathrm{m}$.

Table 2. Intensity of the immunostaining of peroxiredoxins in the human epididymis

\begin{tabular}{|l|c|c|c|c|c|c|}
\hline & PRDX1 & PRDX2 & PRDX3 & PRDX4 & PRDX5 & PRDX6 \\
\hline caput & Pc + & Pc +++ & Pc \pm & Pc + & - & - \\
& $\mathrm{Bc}+$ & & & & \\
\hline corpus & $\mathrm{Pc}+$ & $\mathrm{Pc}+++$ & $\mathrm{Pc} \pm$ & $\mathrm{Pc}+++$ & $\mathrm{Pc} \pm$ & $\mathrm{Pc} \pm$ \\
& $\mathrm{Bc}+$ & & $\mathrm{Bc}+++$ & $\mathrm{Bc}+++$ & $\mathrm{Bc} \pm$ \\
& $\mathrm{Mi}++$ & & & & & $\mathrm{Pc}+$ \\
& $\mathrm{Pc}+++$ & $\mathrm{Pc}+++$ & $\mathrm{Pc} \pm$ & $\mathrm{Pc}+++$ & $\mathrm{Pc} \pm$ & $\mathrm{Bc}+++$ \\
& $\mathrm{Bc}++$ & $\mathrm{Bc}+++$ & $\mathrm{Bc}++$ & $\mathrm{Bc}+++$ & \\
\hline
\end{tabular}

+++ - strong staining; + - moderate staining; \pm - very weak staining; - — no staining. Abbreviations: Pc — principal cells; Bc - basal cells; $\mathrm{St}$ - stereocilia.

staining on the acrosomal region of some sperm heads could still be seen (Fig. 3F). A summary of the immunoreactivities of PRDXs in the human spermatozoa is given in Table 3. There were no qualitative differences in the localization of the PRDXs in the cells of the studied parts of the reproductive tract between subjects. 
Table 3. The immunolocalization of peroxiredoxins in human ejaculated spermatozoa

\begin{tabular}{|l|c|c|c|c|c|c|}
\hline & PRDX1 & PRDX2 & PRDX3 & PRDX4 & PRDX5 & PRDX6 \\
\hline acrosome & - & +++ & - & - & + & +++ \\
\hline equatorial segment & + & - & - & - & - & +++ \\
\hline post-acrosomal region & +++ & - & - & - & - & - \\
\hline neck/cytoplasmic droplet & - & - & - & +++ & - & - \\
\hline midpiece & + & - & + & - & - & - \\
\hline principal piece & - & - & - & - & - & +++ \\
\hline
\end{tabular}

+++ - strong staining; + - moderate staining; - - no staining.

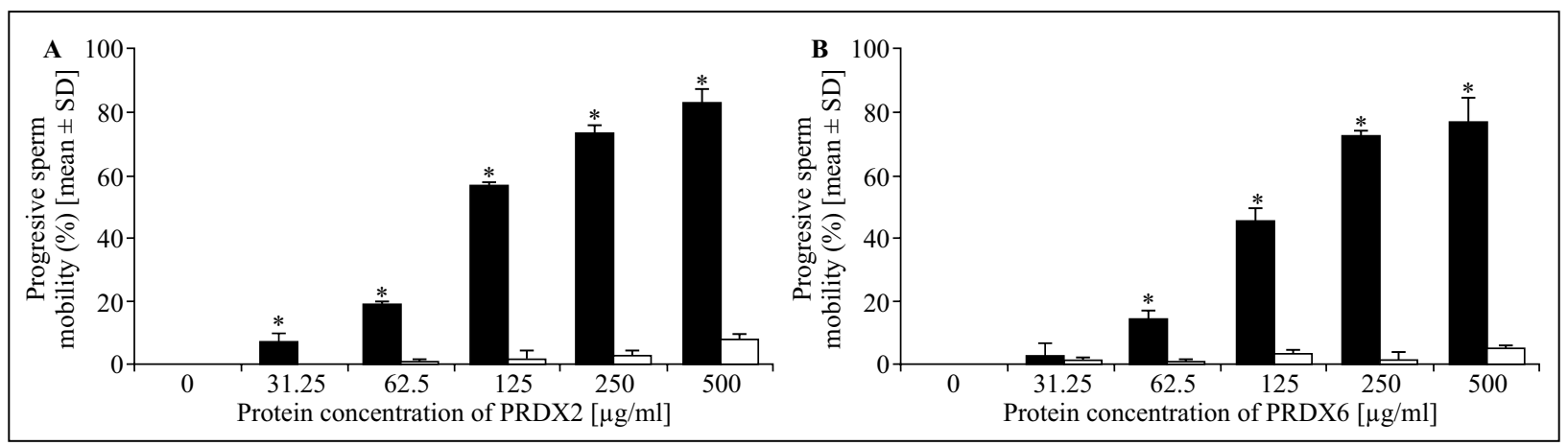

Figure 4. Anti-peroxidative activities of peroxiredoxin (PRDXs) 2 and 6. Sperm motility in the presence of $100 \mu \mathrm{mol} / 1 \mathrm{H}_{2} \mathrm{O}_{2}$ and different concentrations of recombinant PRDX2 (A) and PRDX6 (B). Black columns, PRDXs; blank columns, control cells. *Significantly different from the controls $(\mathrm{P} \leq 0.05)$.

\section{Anti-peroxidative damage activity of PRDX2 and PRDX6}

When $100 \mu \mathrm{mol} / 1 \mathrm{H}_{2} \mathrm{O}_{2}$ was present in the incubation medium no rapidly progressing spermatozoa could be seen. However, simultaneous treatment of spermatozoa with $\mathrm{H}_{2} \mathrm{O}_{2}$ and PRDX2 or PRDX6 significantly increased the percentage motility in a dose-dependent manner, above that of the control group treated with the Trx-His-S tag protein (Fig. 4).

\section{Discussion}

The functions of PRDXs have gained increasing attention since their recognition. O'Flaherty and de Souza showed the existence of PRDX1, 4, 5 and 6 both in spermatozoa and seminal plasma and demonstrated that they were dose-dependently modified by $\mathrm{H}_{2} \mathrm{O}_{2}$, forming disulfide bridges and high molecular mass complexes [20]. Li et al. have shown that all PRDXs are present in human epididymal fluid, and PRDX6 has been shown, as here, to prevent $\mathrm{H}_{2} \mathrm{O}_{2}$-induced sperm motility reduction $[24,25]$. In the present study, the role of PRDX2 and, additionally, PRDX6 in preventing $\mathrm{H}_{2} \mathrm{O}_{2}$-induced sperm motility damage was also demonstrated.

Both spermatogenesis [27] and Leydig cell steroidogenesis $[28,29]$ are vulnerable to oxidative stress and the testis contains an elaborate array of antioxidant enzymes and free radical scavengers that ensure its continuous function. In the present study we detected PRDXs in the human testis, in Sertoli cells, spermatogonia, round spermatids and Leydig cells; results that are consistent with a role for PRDXs in protecting these cells against oxidative stress. Sertoli cells, the supporting cells for germ cells in the testis, offer the main protection against ROS throughout the phase of germ cell development [30, 31]. The current study showed all members of PRDX family to be expressed in Sertoli cells with the staining of PRDX6 particularly intense, suggesting an important protective role for this PRDX in the human testis.

Post-testicular spermatozoa undergo a complex maturation process as they move through the caput and corpus to the cauda of epididymis. Once spermatozoa are released from the germinal epithelium, they are vulnerable to oxidative attack due to many 
reasons such as low level of antioxidant enzymes, their own generation of ROS during capacitation, and their high polyunsaturated fatty acid content [32]. The balance of ROS production and degradation is regulated within cells, and also in the extracellular compartments, through a variety of pathways [32]. The complete cessation of sperm motility in the presence of $\mathrm{H}_{2} \mathrm{O}_{2}$ showed by us in this report and by Li et al. indicates that the intracellular enzymes are insufficiently protective against this non-physiological concentration of ROS [25]. On the other hand, extracellular PRDX6 ([25] and the current study) and PRDX2 (the current study) were able to overcome the $\mathrm{H}_{2} \mathrm{O}_{2}$-induced reduction of motility. This suggests that the extracellular PRDXs of epididymal origin are more effective than the intracellular enzymes in protecting spermatozoa from peroxidative damage under the in vitro conditions. Seminal plasma contains other enzymatic antioxidants (such as SOD, catalase) and a wide range of non-enzymatic antioxidants (such as vitamins $\mathrm{C}$ and $\mathrm{E}$ ) which may also protect spermatozoa against high levels of ROS. However, the mechanisms that underlie the protective activities of the antioxidative enzymes differ from those of vitamins $\mathrm{C}$ and $\mathrm{E}$, which were important chain-breaking antioxidants that prevent sperm agglutination, lipid peroxidation and DNA damage induced by $\mathrm{H}_{2} \mathrm{O}_{2}$ in a dose-dependent manner [33,34]. PRDXs represent a superfamily of Se-independent peroxidases. PRDX2 is a 2-Cys enzyme that uses thioredoxin as an electron donor, whereas PRDX6 is a 1-Cys peroxiredoxin and does not use thioredoxin [35]. We presume that PRDX2 and 6 are important antioxidants in seminal plasma, as shown by their activity against $\mathrm{H}_{2} \mathrm{O}_{2}$ in vitro, but whether they play such a role in vivo needs to be investigated further.

PRDX1 and PRDX2, the two typical 2-Cys PRDXs with Cys47 in the active center, have been considered to serve as efficient ROS sensors in vivo that are involved in dynamic changes in protein structure [36]. The transition between reduced dimers and oligomers, oxidized dimers and hyperoxidized high molecular weight (HMW) assemblies of the enzyme subunits relates to their function, i.e. as a peroxidase, a chaperone, a binding partner or an enzyme activator involved in redox signaling, or a redox sensor. The floodgate theory is that $\mathrm{H}_{2} \mathrm{O}_{2}$ is kept low by PRDX activity in normal cells, whereas PRDX inhibition by hyperoxidation enables $\mathrm{H}_{2} \mathrm{O}_{2}$ signaling [37]. In the current study, there was obvious expression of PRDX1, 2, 4 and 6 in the epididymal principal cells and we speculate that PRDXs, in particular the typical 2-Cys PRDX1 and PRDX2 present in high amounts in the stereocilia bordering the tubule lumen, may play a sensor role, responding in activity to the ROS level of the human epididymal microenvironment for sperm maturation and storage. This hypothesis receives support from studies in the rat, in which, caput epididymidal spermatozoa presented significantly higher PHGPx, GPx and SOD activities, and a greater content of vitamin $\mathrm{E}$ than cauda epididymidal spermatozoa [38]. The consequent higher risk of oxidative damage to cauda spermatozoa may be compensated by the microenvironment which controls ROS generation due to the presence of the antioxidant enzymes [38]. As the present study showed that PRDXs exist widely in epididymal epithelial cells, and the expressions of PRDX1, 4, 5, 6 were higher in the cauda than in the caput and corpus, this mechanism may operate in man. We propose that PRDXs are potential antioxidant protectors for human cauda epididymidal spermatozoa.

Although most germ cell cytoplasm has been discarded by the time spermatozoa are discharged into the lumen of the seminiferous tubules, there are remains confined to the neck in the form of the cytoplasmic droplet, in the vicinity of the mitochondria. In somatic cells PRDX1 is located in the cytoplasm and nucleus [9] and PRDX3 and 5 are localized to the mitochondrial matrix $[39,40]$. Our observations suggests that sperm PRDX1 is more likely to be localized in the cytoplasm between mitochondria and plasma membrane than in the mitochondria, whereas the mitochondrial PRDX3, but not PRDX5, is localized in the midpiece of the sperm tail. PRDX3 was located at the site of ROS production, and may prevent the accumulation of ROS to the levels we used in vitro, that overwhelmed this protective capability.

Interestingly, every sperm compartment contained at least one PRDX, giving the sperm cell full-cover protection. Most regions of the spermatozoon, such as the acrosome, equatorial segment, neck, and principal piece, may be protected by the PRDX2, 4, 5 and 6 found there, as shown in other species [17-21]. Whereas the presence of PRDX2, 3 in human spermatozoa was a novel finding in this report' the localization of the other PRDXs mostly agree with those reported by O'Flaherty and de Souza [20], except for PRDX4. Our finding of PRDX4 presence in the neck and cytoplasmic droplet, the remains of spermatid cytoplasm in human ejaculated spermatozoa, is consistent with the finding in the mouse [18] in which PRDX4 is present in the elongating spermatid cytoplasm and in the residual bodies representing the cytoplasm eliminated before sperm release.

The localization of PRDX6 in male reproductive tract reported here differs from that observed previously [25], as a new antibody was used. The eliciting 
of a new antibody was necessary since the earlier antibody, raised to an internal region of the protein, was found to have lost its immunogenicity to the fulllength fusion proteins. The rabbit anti-human PRDX6 antibody used in the current study was produced against the full-length recombinant protein, and its specificity was verified by immunoblotting with the commercial human PRDX6 recombinant protein.

In conclusion, we present here a systemic study of the localization of all the family members of PRDXs in the human testis, epididymis and spermatozoa. The obtained results suggest that PRDX2 and 6 play an important role in protecting spermatozoa from peroxidative damage in vitro and possibly also in vivo.

\section{Acknowledgments}

This research was financially supported by the grants from the National Natural Science Foundation of China (Grant No. 81300738, 30271213 and 81200432). We thank Dr. Trevor G. Cooper and Dr. Ching-Hei Yeung for critical review and editing of the manuscript.

\section{References}

1. Gagnon C, Iwasaki A, De Lamirande E, et al. Reactive oxygen species and human spermatozoa. Ann N Y Acad Sci. 1991; 637: 436-444, indexed in Pubmed: 1785786.

2. Garg A, Kumaresan A, Ansari MR. Effects of hydrogen peroxide (H2O2) on fresh and cryopreserved buffalo sperm functions during incubation at 37 degrees $\mathrm{C}$ in vitro. Reprod Domest Anim. 2009; 44(6): 907-912, doi: 10.1111/j.14390531.2008.01115.x, indexed in Pubmed: 18992123.

3. Saleh R, Agarwal A, Kandirali E, et al. Leukocytospermia is associated with increased reactive oxygen species production by human spermatozoa. Fertility and Sterility. 2002; 78(6): 1215-1224, doi: 10.1016/s0015-0282(02)04237-1.

4. Sikka SC. Oxidative stress and role of antioxidants in normal and abnormal sperm function. Front Biosci. 1996; 1: e78-e86, indexed in Pubmed: 9159248.

5. Maneesh M, Jayalekshmi H, Dutta S, et al. Effect of chronic ethanol administration on testicular antioxidant system and steroidogenic enzyme activity in rats. Indian J Exp Biol. 2005; 43(5): 445-449, indexed in Pubmed: 15900910.

6. Padron OF, Brackett NL, Sharma RK, et al. Seminal reactive oxygen species and sperm motility and morphology in men with spinal cord injury. Fertil Steril. 1997; 67(6): 1115-1120, indexed in Pubmed: 9176453.

7. Gong S, San Gabriel MC, Zini A, et al. Low amounts and high thiol oxidation of peroxiredoxins in spermatozoa from infertile men. J Androl. 2012; 33(6): 1342-1351, doi: 10.2164/ /jandrol.111.016162, indexed in Pubmed: 22492841.

8. Chae HZ, Kim HJ, Kang SW, et al. Characterization of three isoforms of mammalian peroxiredoxin that reduce peroxides in the presence of thioredoxin. Diabetes Res Clin Pract. 1999; 45(2-3): 101-112, indexed in Pubmed: 10588361.

9. Wood ZA, Schröder E, Robin Harris J, et al. Structure, mechanism and regulation of peroxiredoxins. Trends Biochem Sci. 2003; 28(1): 32-40, indexed in Pubmed: 12517450.

10. Murphy MP. How mitochondria produce reactive oxygen species. Biochem J. 2009; 417(1): 1-13, doi: 10.1042/BJ20081386, indexed in Pubmed: 19061483.
11. Anwar Sh, Yanai T, Sakai H. Overexpression of Peroxiredoxin 6 Protects Neoplastic Cells against Apoptosis in Canine Haemangiosarcoma. J Comp Pathol. 2016; 155(1): 29-39, doi: 10.1016/j.jcpa.2016.05.002, indexed in Pubmed: 27306414.

12. Rahman MdS, Kwon WS, Lee JS, et al. Bisphenol-A affects male fertility via fertility-related proteins in spermatozoa. Sci Rep. 2015; 5: 9169, doi: 10.1038/srep09169, indexed in Pubmed: 25772901.

13. Sutovsky P, Aarabi M, Miranda-Vizuete A, et al. Negative biomarker based male fertility evaluation: Sperm phenotypes associated with molecular-level anomalies. Asian J Androl. 2015; 17(4): 554-560, doi: 10.4103/1008-682X.153847, indexed in Pubmed: 25999356.

14. Lee D, Moawad AR, Morielli T, et al. Peroxiredoxins prevent oxidative stress during human sperm capacitation. Mol Hum Reprod. 2017; 23(2): 106-115, doi: 10.1093/molehr/gaw081, indexed in Pubmed: 28025393.

15. Ozkosem B, Feinstein S, Fisher A, et al. Absence of Peroxiredoxin 6 Amplifies the Effect of Oxidant Stress on Mobility and SCSA/CMA3 Defined Chromatin Quality and Impairs Fertilizing Ability of Mouse Spermatozoa. Biology of Reproduction. 2016; 94(3), doi: 10.1095/biolreprod.115.137646.

16. Kwon WS, Rahman MdS, Lee JS, et al. A comprehensive proteomic approach to identifying capacitation related proteins in boar spermatozoa. BMC Genomics. 2014; 15: 897, doi: 10.1186/1471-2164-15-897, indexed in Pubmed: 25315394.

17. Dammeyer P, Arnér ESJ. Human Protein Atlas of redox systems - what can be learnt? Biochim Biophys Acta. 2011; 1810(1): 111-138, doi: 10.1016/j.bbagen.2010.07.004, indexed in Pubmed: 20647035.

18. Godoy J, Funke M, Ackermann W, et al. Redox atlas of the mouse. Biochimica et Biophysica Acta (BBA) - General Subjects. 2011; 1810(1): 2-92, doi: 10.1016/j.bbagen.2010.05.006.

19. Fujii T, Fujii J, Taniguchi N. Augmented expression of peroxiredoxin VI in rat lung and kidney after birth implies an antioxidative role. Eur J Biochem. 2001; 268(2): 218-225, indexed in Pubmed: 11168354.

20. O'Flaherty C, Souza AR. Hydrogen Peroxide Modifies Human Sperm Peroxiredoxins in a Dose-Dependent Manner. Biology of Reproduction. 2010; 84(2): 238-247, doi: 10.1095/ /biolreprod.110.085712.

21. van Gestel RA, Brewis IA, Ashton PR, et al. Multiple proteins present in purified porcine sperm apical plasma membranes interact with the zona pellucida of the oocyte. Mol Hum Reprod. 2007; 13(7): 445-454, doi: 10.1093/molehr/gam030, indexed in Pubmed: 17483085.

22. Shi H, Yu HJ, Wang HY, et al. Topical administration of peroxiredoxin- 6 on the cornea suppresses inflammation and neovascularization induced by ultraviolet radiation. Invest Ophthalmol Vis Sci. 2012; 53(13): 8016-8028, doi: 10.1167/ /iovs.12-10064, indexed in Pubmed: 23139277.

23. Lin YQ, Li JY, Wang HY, et al. Cloning and identification of a novel sperm binding protein, HEL-75, with antibacterial activity and expressed in the human epididymis. Hum Reprod. 2008; 23(9): 2086-2094, doi: 10.1093/humrep/den084, indexed in Pubmed: 18343807.

24. Li J, Liu F, Liu X, et al. Mapping of the human testicular proteome and its relationship with that of the epididymis and spermatozoa. Mol Cell Proteomics. 2011; 10(3): M110.004630, doi: 10.1074/mcp.M110.004630, indexed in Pubmed: 21178120.

25. Li J, Liu F, Wang H, et al. Systematic mapping and functional analysis of a family of human epididymal secretory sperm-located proteins. Mol Cell Proteomics. 2010; 9(11): 2517-2528, doi: 10.1074/mcp.M110.001719, indexed in Pubmed: 20736409. 
26. Bradford MM. A rapid and sensitive method for the quantitation of microgram quantities of protein utilizing the principle of protein-dye binding. Anal Biochem. 1976; 72: 248-254, indexed in Pubmed: 942051.

27. Peltola V, Mäntylä E, Huhtaniemi I, et al. Lipid peroxidation and antioxidant enzyme activities in the rat testis after cigarette smoke inhalation or administration of polychlorinated biphenyls or polychlorinated naphthalenes. J Androl. 1994; 15(4): 353-361, indexed in Pubmed: 7982804.

28. Quinn PG, Payne AH. Oxygen-mediated damage of microsomal cytochrome P-450 enzymes in cultured leydig cells. Role in steroidogenic desensitization. J Biol Chem 1984; 259:41304135. ...nih. https://www ncbi nlm gov/pubmed/? term =J + Biol+Chem+1984\%3B. ; 259: 3A4130-4135.

29. Chen H, Liu J, Luo L, et al. Vitamin E, aging and Leydig cell steroidogenesis. Exp Gerontol. 2005; 40(8-9): 728-736, doi: 10.1016/j.exger.2005.06.004, indexed in Pubmed: 16054318.

30. Bauché F, Fouchard MH, Jégou B. Antioxidant system in rat testicular cells. FEBS Lett. 1994; 349(3): 392-396, indexed in Pubmed: 8050602.

31. BOER PJ, POOT M, VERKERK A, et al. Glutathione-dependent defence mechanisms in isolated round spermatids from the rat. International Journal of Andrology. 1990; 13(1): 26-38, doi: 10.1111/j.1365-2605.1990.tb00957.x.

32. Aitken RJ, Curry BJ. Redox regulation of human sperm function: from the physiological control of sperm capacitation to the etiology of infertility and DNA damage in the germ line. Antioxid Redox Signal. 2011; 14(3): 367-381, doi: 10.1089/ /ars.2010.3186, indexed in Pubmed: 20522002.

33. Makker K, Agarwal A, Sharma R. Oxidative stress \& male infertility. Indian J Med Res 2009; 129: 357-367, indexed in Pubmed: 19535829.
34. Kodama H, Yamaguchi R, Fukuda J, et al. Increased oxidative deoxyribonucleic acid damage in the spermatozoa of infertile male patients. Fertility and Sterility. 1997; 68(3): 519-524, doi: 10.1016/s0015-0282(97)00236-7.

35. Manevich Y, Fisher AB. Peroxiredoxin 6, a 1-Cys peroxiredoxin, functions in antioxidant defense and lung phospholipid metabolism. Free Radic Biol Med. 2005; 38(11): 1422-1432, doi: 10.1016/j.freeradbiomed.2005.02.011, indexed in Pubmed: 15890616 .

36. Nicolussi A, D'Inzeo S, Capalbo C, et al. The role of peroxiredoxins in cancer. Mol Clin Oncol. 2017; 6(2): 139-153, doi: 10.3892/mco.2017.1129, indexed in Pubmed: 28357082.

37. Barranco-Medina S, Lázaro JJ, Dietz KJ. The oligomeric conformation of peroxiredoxins links redox state to function. FEBS Lett. 2009; 583(12): 1809-1816, doi: 10.1016/j. febslet.2009.05.029, indexed in Pubmed: 19464293.

38. Tramer F, Rocco F, Micali F, et al. Antioxidant Systems in Rat Epididymal Spermatozoa1. Biology of Reproduction. 1998; 59(4): 753-758, doi: 10.1095/biolreprod59.4.753.

39. Kropotov A, Usmanova N, Serikov V, et al. Mitochondrial targeting of human peroxiredoxin $\mathrm{V}$ protein and regulation of PRDX5 gene expression by nuclear transcription factors controlling biogenesis of mitochondria. FEBS J. 2007; 274(22): 5804-5814, doi: 10.1111/j.1742-4658.2007.06103.x, indexed in Pubmed: 17937766.

40. Knoops B, Clippe A, Bogard C, et al. Cloning and characterization of AOEB166, a novel mammalian antioxidant enzyme of the peroxiredoxin family. J Biol Chem. 1999; 274(43): 30451-30458, indexed in Pubmed: 10521424.

Submitted: 30 May, 2018

Accepted after reviews: 10 August, 2018 Available as AoP: 30 August, 2018 